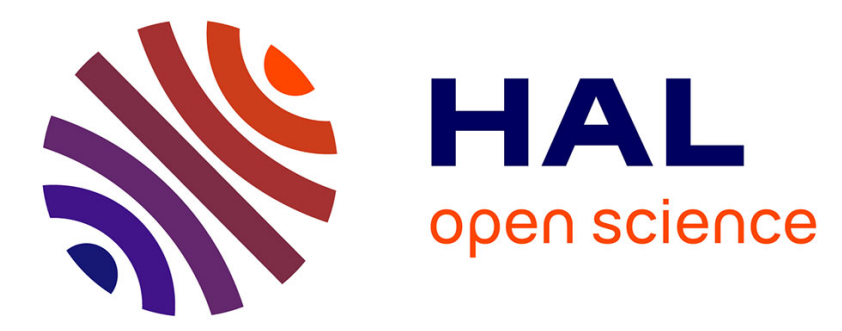

\title{
Producción de material didáctico para la salud de las comunidades amerindias del Amazonas venezolano
}

Jean Chiappino

\section{To cite this version:}

Jean Chiappino. Producción de material didáctico para la salud de las comunidades amerindias del Amazonas venezolano. Caminos Cruzados. Ensayos en Antropología Social, Etnoecología y Etnoeducación, C. Alès \& J. Chiappino (dir.): 563-580. Mérida, IRD Editions/ULA-GRIAL, 2003. halshs-02546651

\section{HAL Id: halshs-02546651 \\ https://shs.hal.science/halshs-02546651}

Submitted on 3 May 2020

HAL is a multi-disciplinary open access archive for the deposit and dissemination of scientific research documents, whether they are published or not. The documents may come from teaching and research institutions in France or abroad, or from public or private research centers.
L'archive ouverte pluridisciplinaire HAL, est destinée au dépôt et à la diffusion de documents scientifiques de niveau recherche, publiés ou non, émanant des établissements d'enseignement et de recherche français ou étrangers, des laboratoires publics ou privés. 
Caminos Cruzados. Ensayos en Antropología Social, Etnoecología y Etnoeducación, C. Alès \& J. Chiappino (dir.): 563-580. Mérida, IRD Editions/ULA-GRIAL, 2003.

\title{
Producción de material didáctico para la salud de las comunidades amerindias del Amazonas venezolano
}

\author{
Jean Chiappino*
}

La obtención de la participación de las comunidades en el control de la salud constituye una de las mayores dificultades que encuentra el sistema sanitario planteado para los pueblos autóctonos. La biomedicina, por sí sola, no puede luchar en contra de las endemias sin la estricta observación, por parte de la población, de imprescindibles conductas terapéuticas y profilácticas. La tasa de las fiebres maláricas, por ejemplo, no puede retroceder si los enfermos, por un lado, no se tratan correctamente y siguen siendo portadores del Plasmodium -parásito responsable de la malaria-, y si el pueblo, por otro lado, no toma las medidas necesarias para luchar contra la propagación del zancudo, vector de transmisión de esta enfermedad.

Los médicos, enfermeros y auxiliares de salud del estado Amazonas, caso del cual hablaremos, saben bien que la falta de comprensión de las estrategias biomédicas por las comunidades es una de las causas que debe enfrentar el dispositivo de atención sanitaria. La inteligibilidad de las prescripciones médicas constituye para ellos, con el abastecimiento en remedios de los ambulatorios, una condición fundamental para que la presencia médica no siga siendo vana ${ }^{1}$.

\footnotetext{
* Institut de Recherche pour le Développement (IRD), CELIA (CNRS), Paris.
} 
Tomando en cuenta esta situación, se planificó un programa de investigación-acción principalmente basado sobre la aproximación de las ciencias sociales ${ }^{2}$. Su propósito consistía en elaborar un plan de educación sanitaria de los pueblos amerindios del Amazonas venezolano, focalizado en la comunicación de un mensaje médico claramente comprensible por las poblaciones ${ }^{3}$.

En este artículo trataremos de la producción de folletos bilingües ${ }^{4}$ que se realizaron en el marco de este programa y, principalmente, de los aspectos relativos a la traducción de los preceptos biomédicos. Veremos que estos documentos responden a la aspiración de las comunidades en participar en el control de su salud. Constituyen unos de los instrumentos útiles para que se realice la necesaria colaboración entre las comunidades y el servicio médico-asistencial y se mejore el estado de salud de las poblaciones indígenas. Facilitando la comprensión de la acción médica, estimulan la participación de los pueblos en el control de las endemias. En su versión en lengua autóctona, representan un medio de comunicación susceptible de compensar la dificultad del personal de salud indígena del Servicio de Medicina Simplificada para transmitir prácticas que, en muchos casos, ellos mismos reconocen no entender muy bien. En su versión castellana, constituyen un modelo de expresión que puede ayudar a los médicos para ser comprendidos por los enfermos y agentes de salud indígenas, en la formación de los cuales ellos quieren colaborar activamente. En realidad, los indígenas capacitados conforman un personal local muy capaz de servirles en el desarrollo sanitario de las comunidades en las cuales ellos conviven de manera más continua que el personal exógeno.

\section{Condiciones previas a la traducción de los folletos}

La producción de los folletos dedicados a reforzar la salud de los pueblos indígenas del estado Amazonas necesitó traducir y adaptar los preceptos médicos referentes a las mayores endemias presentes. A partir de la evaluación realizada por la Dirección Regional de Salud del estado se decidió considerar, en primer lugar, las enfermedades endémicas más graves para el porvenir de las poblaciones y los temas higiénicos necesaria- 
mente complementarios. Se seleccionó así las diarreas infantiles, la malaria, la tuberculosis, las infecciones respiratorias agudas de los niños, las enfermedades sexualmente transmisibles y el SIDA, los problemas vinculados con el agua de beber, así como la higiene en general. Conforme a las dificultades vinculadas con las 19 lenguas habladas en la región (ver OCEI 1993), el programa se concentró en la traducción de las prescripciones médicas en las lenguas de los grupos numéricamente mas importantes como los Yanomami, los Wothïha (Piaroa), los Hiwi (Guahibo), los Ye'kwana (Makiritare), los Kurripako y los Chase (Piapoco).

Dos objetivos guiaron el trabajo de producción de los documentos. Dar prioridad a la inteligibilidad del mensaje médico, y evitar la descalificación de las prácticas tradicionales así como la sobre-evaluación de la medicina moderna.

La comprensión de las conductas terapéuticas y profilácticas occidentales por las comunidades depende de la traducción del mensaje biomédico, pero ésta no puede considerarse como una traducción literal de las recetas prescritas por los médicos ni tampoco limitarse, como se suele pensar, a una simple transposición de ellas al lenguaje coloquial.

Para que el enfermo, indígena -o no-, hablante del castellano -o no-, siga el tratamiento y, por eso, frecuente debidamente el ambulatorio, se necesita que entienda la obligación de modificar su comportamiento habitual. Esta aceptación depende de todo un contexto socio-cultural que el mensaje médico debe integrar en el acto comunicativo que constituye la consulta. La transmisión de explicaciones es indispensable para que los elementos del diagnóstico y las prescripciones médicas sean aceptadas. Esta condición es sumamente importante para el médico o el enfermero a pesar de la dificultad inherente a la situación lingüística. En un caso de malaria o de cualquier otra enfermedad endémica, se entiende que la toma forzada de un comprimido que debe tragar el enfermo, gesto al cual puede considerarse que se reduce el acto médico en una situación de incomunicabilidad, no basta para curar el enfermo ni para resolver la situación mórbida. Además, provoca generalmente una resistencia a la medicina moderna. En la práctica médica, la comunicación forma parte de la relación que todo agente sanitario debe mantener con el enfermo y, mu- 
chas veces, con sus familiares para que sean aceptadas las medidas indispensables para el establecimiento del diagnóstico, la terapia y/o la profilaxis. El texto de los folletos trata entonces de presentar todos los argumentos contextuales y socio-culturales necesarios para el entendimiento no sólo de la receta prescrita, sino también de los exámenes y controles indispensables para la cura de la enfermedad.

\section{Traducción de los folletos}

La transposición y la traducción de los textos suponen un trabajo pluri-disciplinario. Resultan, en primer lugar, de las encuestas antropológicas organizadas para entender la concepción de las sociedades amerindias acerca del problema sanitario escogido, así como su manera de pensar la enfermedad y la salud en general.

En nuestro caso, este trabajo, además de haber beneficiado del avance de las investigaciones y análisis ya realizados en relación con esta materia, ha consistido en una primera fase en recopilar datos mediante talleres 5 que reunían tanto al personal médico-asistencial como a los representantes de las comunidades durante 2 a 3 días. Repartidos en mesas según las etnias, los participantes reflexionan sobre la concepción particular que ellos mismos tienen de la enfermedad o del problema sanitario estudiado en el taller. Luego cada grupo de trabajo presenta públicamente sus resultados y un especialista expone el punto de vista de la medicina moderna. Sigue un debate y una confrontación de ideas.

A continuación de los talleres se redacta un primer texto a partir, de un lado, de los datos biomédicos proporcionados por los médicos y, por otro lado, de los datos antropológicos recogidos por los antropólogos a fin de adaptar el mensaje médico básico. La redacción del texto es sometida a dos exigencias, conformarse con la prescripción determinada por el Ministerio de Salud para el problema sanitario considerado e integrar principios autóctonos fundamentales. La toma en cuenta de tales condiciones da mayores posibilidades de lograr un mejor control de la salud, pues satisface tanto a la lógica biomédica como a las leyes culturales propias de cada sociedad. Las recomendaciones serán aun más 
aceptadas por las poblaciones indígenas que se dialoga con sus normas socio-culturales.

En esta primera fase, el texto es el resultado de un trabajo de adaptación al universo amerindio de un problema sanitario tratado desde el punto de vista biomédico. Contiene además de los preceptos médicos relativos al diagnóstico, tratamiento y profilaxis de cada enfermedad, un breve recuento histórico de la evolución del pensamiento occidental y un corto relato que valoriza el saber de la sociedad indígena sobre el tema.

Se observa que la indicación de la variación con el tiempo de la concepción médica sobre las afecciones es una realidad muy bien recibida por los pueblos indígenas. El hecho de saber que el conocimiento médico actual no fue adquirido de una vez pero en el transcurso de un largo proceso histórico, relativiza la impresión todopoderosa de la medicina occidental y le confiere una accesibilidad más inmediata. Además, el conocimiento de las concepciones antiguas que nuestros padres tenían acerca del tema sanitario y que no se encuentran en muchos casos tan alejadas de las que tienen las culturas amerindias, facilita la aceptación de las estrategias actuales que se trata de enseñarles.

En una segunda fase, se considera la propia traducción del texto. La primera versión castellana de la cartilla se somete a prueba en algunas comunidades para averiguar el grado de comprensión del texto, contando para ello con locutores bilingües de cada cultura, cuando es posible.

Como consecuencia de esta consulta se mejora el texto al descubrir puntos de incomprensión. Éste sigue mejorándose y complementándose a lo largo de su traducción en las diversas lenguas, al comparar las reacciones de los traductores. Estas mejoras y complementos explicativos implican revisar la versión castellana del texto original.

La dificultad que representa el deseo de traducir à la carte, para cada grupo étnico y conforme a su manera de concebir la enfermedad y la salud, los preceptos biomédicos y la complejidad de sus estrategias no es sino aparente. Depende, en buena parte, de la costumbre que tenemos de oponer de manera radical la biomedicina y las prácticas de las sociedades amerindias. La representación materialista que tenemos, por un lado, de la medicina moderna, que reducimos a la teoría alopática de la sanación, y 
la representación espiritualista que tenemos, por otro lado, de los comportamientos sanitarios amerindios -que focalizamos sobre los aspectos más extraños de la actividad chamánica-, nos impiden ver los puntos de acercamiento lógico existentes entre ambas técnicas medicinales (ver J. Chiappino 1995a, 1995b, 1997).

La representación que las culturas amerindias elaboraron a propósito de la materia viva y su reproducción nos ofrece conceptos muy adecuados para dar cuenta de la potencia de los remedios propios de la medicina alopática. Aunque los términos generalmente empleados para traducir estos conceptos, tal como los de «alma» o de "espíritu», refieren al orden sagrado, se recomienda utilizar las palabras de «fuerza vital» o «energía» que se avienen perfectamente en el contexto biomédico que nos concierne. Por otra parte, las culturas amerindias valorizan un principio de equilibrio isomórfico que se manifiesta en la sobrevivencia de la materia viva y que está asociado a un complejo paradigma de oposiciones de categorías tales como caliente/frío, dulce/salado, seco/húmedo, alto/bajo y vivo/muerto (ver A. Butt Colson y C. Armellada 1977, 1985; J. Clarac de Briceño 1981; J. Chiappino 1995 a, 1995b). Estas analogías permiten volver inteligibles, por ejemplo, las técnicas de rehidratación relativas a las diarreas, pero también numerosos principios de lucha en contra de los zancudos -caso de la malaria- u otras enfermedades vinculadas con vectores (ver J. Chiappino 1997). La práctica tradicional de lixiviación, por ejemplo, que interviene en el enfriamiento de la yuca, así como las estrategias de búsqueda de las enfermedades por los espíritus, nos ofrecen muchas comparaciones posiblemente explotables para explicar ciertos medios de diagnóstico o de profilaxis como las vacunas en el caso, entre otros, de la tuberculosis (J. Chiappino ibid.).

La conformidad de los textos con la lógica médica de las prescripciones médicas, depende de la habilidad de los traductores para entender el mensaje y con ello lograr adaptarlo al modo de pensar autóctono sin desviarlo ni inducir a equívocos, sobre todo, en la dosis y duración del tratamiento. No obstante, la adecuación de los textos a la lógica médica no impone obstáculos insuperables. El concepto de energía o fuerza, y las concepciones relativas al equilibrio vital convienen, con el apoyo de analo- 
gías chamánicas, para cubrir los campos conceptuales inherentes al propósito educativo que planteamos. No se necesita, como sucede en otros contextos, crear conceptos para traducir nociones inexistentes ${ }^{6}$. El conocimiento antropológico tanto de la lógica del acto médico como de las prácticas chamánicas permite reinterpretar el discurso generalmente demasiado elitista de la medicina moderna para elaborar unos folletos de salud que sean a la vez inteligibles, adaptados a las posibilidades de recepción de las poblaciones y que no transmitan malentendidos, cuyas consecuencias son bastante graves para la comprensión y la eficacia de la acción médica.

\section{Adaptación de las prescripciones médicas a la cultura amerindia}

En la producción de folletos para la salud de las comunidades indígenas la traducción del mensaje biomédico corresponde a algo diferente que la pura transposición lingüística: abarca con una profunda atención a las características culturales de las comunidades a las cuales se dirige. Aunque sean elaboradas para enseñar prácticas nuevas, el mensaje que transmiten no considera solamente la comprensión de técnicas y estrategias. Debe demostrar que la acción médica no se opone a los valores fundamentales de la cultura ni trata de sustituirlos.

Se necesita, aquí, recordar que para muchas comunidades del Amazonas, estas nuevas prácticas son desconocidas. Varias veces son consideradas capaces de transformar el ser e, incluso, cargadas de una intención potencialmente nociva.

En el caso de la diarrea del niño, por ejemplo, hablar de hacer tomar una gran cantidad de agua y de sal para explicar la acción del suero oral de rehidratación, es un procedimiento que las familias pueden pensar como dañino. En la concepción amerindia de la vida, la toma de agua con sal se concibe como un exceso de frío que puede oponerse al equilibrio vital de la persona ${ }^{7}$. El elemento hídrico y el elemento salino son ambos del orden del frío y pueden agravar el equilibrio vital del niño ya perturbado por el agente causal de la diarrea. De allí el rechazo del suero oral cuando está solamente presentado con la expresión inspirada de la fórmu- 
la occidental «dar de beber mucha agua con sal». El malentendido al origen de este rechazo toma toda su importancia cuando uno se recuerda que la diarrea infantil es una de las mayores causas de muerte entre esas poblaciones $^{8}$. En estos casos, y sin entrar en los detalles de la explicación de la presión hidrostática interesada en la curación de la diarrea, es suficiente comparar el suero oral a las papillas autóctonas utilizadas para curar dicho desequilibrio. En esta perspectiva se recomienda utilizar la palabra "tés" para explicar el efecto del suero oral, comparándolo así al efecto «suavizante» de las hierbas tradicionalmente empleadas en contra de la diarrea. Así se da a entender muy claramente la potencialidad del suero oral sin contradecir las concepciones indígenas según las cuales toda ruptura brutal y/o prolongada del equilibrio vital del cuerpo puede llevar al individuo a la muerte.

También un rechazo de las vacunas o de los remedios puede ocurrir si se sugiere que la vacuna equivale a la introducción en el cuerpo de agentes extraños. En este caso, las poblaciones pueden percibir una voluntad de transformar a la persona. Mejor entonces explicar la estrategia de «suavización» empleada en la preparación de las vacunas y compararla con el «lavado» de la yuca.

Por cierto, un mensaje que contiene argumentos contrarios a los principios fundamentales de la sociedad no facilita la acción médica. No obstante, la resistencia a la toma del remedio o del seguimiento de los tratamientos o inyecciones profilácticas (las vacunas), así como el rechazo del dispositivo de salud planteado para las comunidades indígenas, no dependen exclusivamente de malentendidos y desacuerdos relativos a los principios fisiológicos fundamentales para la sociedad. Son también función del desconocimiento de los principios fundamentales vinculados con el orden social y simbólico.

La falta de reconocimiento de los chamanes y la poca atención acordada al papel de los mayores y de las personas influyentes de las sociedades amerindias -las que son sabias y guían a las comunidades- refuerzan la resistencia y rechazo acerca de la intervención médica.

El caso ocurre en los territorios indígenas cuando el personal asistencial, concentrado sobre el único aspecto biológico (caso del MSAS, 
ver MSAS 1991) y/o conmovido por una ideología religiosa (sobre ese punto, Chiappino 1992), desvaloriza el conocimiento ancestral y descalifica los poderes de los especialistas indígenas.

A fin de lograr la aceptación, a la vez, de las recomendaciones sobre los nuevos comportamientos y sobre el personal que los transmite, el texto de los folletos buscará evitar de presentar una ideología capaz de introducir rupturas sociales. En este sentido no es muy adecuado que el servicio médico, que se propone ayudar y reforzar la salud de las comunidades, condene la actividad chamánica.

No hay obligación ninguna de presentar las técnicas de la medicina occidental como exclusivas de las prácticas ancestrales, y mucho menos cuando se sabe que se debe aprovechar, en muchas oportunidades, remedios y técnicas curativas autóctonos como consecuencia de la escasez de remedios en los ambulatorios. El dispositivo oficial no puede tampoco, por su parte, concebirse como autónomo en cuanto a la selección del personal local que incorpora y al papel social que éste tiene que cumplir a través de su cargo, cuando se reconoce que, por falta de personal, se necesita la presencia de personas autóctonas. Con estas condiciones ¡cómo pensar que el servicio sanitario se puede dispensar sin obtener el consenso previo de los pueblos, tanto sobre el mensaje médico y el personal local que lo va a transmitir? ¿Cómo pensar que un sistema de salud que daría la impresión de introducir unas graves fracturas sociales a propósito de la concepción del ser humano, el orden social y/o cósmico pueda ser aceptado y pueda provocar la participación de las comunidades en el control de la salud?

En este contexto la producción de los folletos supone, además de la evaluación de su traducción por parte de los médicos para que se conforme a la lógica científica propia de la medicina, una necesaria evaluación por parte de los chamanes y ancianos para que el texto sea conforme a los principios fundamentales propios no sólo de su concepción de la enfermedad y la salud, sino también de la organización social.

La adecuación de los textos a la estructura social y al papel del hombre en el universo, depende de la manera cómo se incluye referencias al lado de nuevas estrategias sanitarias a la función de los chamanes y al conocimiento de los ancianos y ancestros. El propósito del mensaje no 
consiste en enseñar el saber local ni tampoco la terapéutica chamánica. Consiste en reconocer los conocimientos y valores indígenas a dentro de la técnicas médicas enseñadas.

La consulta de los chamanes y ancianos acerca del texto constituye ya un primer reconocimiento de la organización social. El visto bueno por parte de estos personajes facilita la aceptación así como el seguimiento de las recomendaciones que los jóvenes auxiliares de salud transmitirán.

Las referencias al conocimiento tradicional y ancestral pueden variar según la enfermedad y la sociedad. Cuando se trata, por ejemplo, de la preparación del suero oral de rehidratación del niño con diarrea aguda, se recomienda a los grupos en los cuales se necesita que el chamán «sople» toda nueva comida del niño, que lo haga sobre el agua que sirve para preparar el suero. Se aconseja también, cuando la comunidad no posea los sobres de sales para confeccionar tal suero y en la espera de la llegada de los sobres que hay que mandar a buscar rápidamente, que se utilice los tés o bien las papillas que se preparan tradicionalmente para estos casos. Esto mismo se puede realizar para cualquier toma de remedio.

En las sociedades donde los rezos de un chamán son la costumbre, se aconseja asociarlos con la toma de la prescripción médica. La intervención del médico o del servicio médico, así como el uso del remedio, no deben prohibir la actuación de un chamán si el grupo lo desea. Ninguna de esas prácticas entran en contradicción radical con la lógica biomédica ni la prohiben cuanto más claramente sea explicada y argumentada. Hemos observado repetidamente que, cualquiera sea el veto que se oponga, la actuación de los chamanes de todos modos sigue realizándose conjuntamente cuando la comunidad la desea, ya sea de manera visible o a escondidas. Seguir descalificando y/o prohibiendo los actos chamánicos no logra sino provocar el desinterés hacia la medicina. Si bien las comunidades indígenas del Amazonas reconocen una eficacia aparente a la medicina moderna, no se la acreditan la eficiencia necesaria para resolver el problema mórbido. Según sus concepciones, la enfermedad es frecuentemente asociada con una intencionalidad nociva de un ser humano o de un ser sobrenatural y la resolución del mal requiere la intervención de un chamán. $\mathrm{Al}$ sistema etiológico autóctono corresponde un sistema terapéutico del 
cual la biomedicina no es representativa y al cual no puede sustituirse. A fuerza de una lógica de dominación cultural, religiosa o política, la actividad chamánica no entra en contradicción con la lógica científica de la biomedicina. Del punto de vista de la práctica médica ella está aún recomendada si contribuye al bienestar del enfermo y de su familia.

\section{Adaptaciones relativas a la comprensión de la dosis $y$ duración del tratamiento occidental}

En la adaptación de la traducción al componente socio-cultural, surge también el complejo problema de la comprensión de la sanación en relación con la duración del tratamiento y la repartición cotidiana del remedio. Para la dificultad de memorización de las tomas de remedios, su repartición en el día y la duración total del tratamiento se puede recurrir a una representación gráfica.

Debemos subrayar que la linealidad empleada para representar, en nuestra sociedad, una secuencia con un esquema dibujado para ser leído siguiendo un rumbo que va de la izquierda a la derecha y de arriba hacia abajo, no es sistemáticamente significativo para la mayoría de los casos amerindios.

Al nivel lexical, la secuencia del tratamiento en el día se traduce refiriéndose muy sencillamente a las principales posiciones del sol -amanecer, mediodía, atardecer- y su duración, a las posiciones de la luna. Al nivel ilustrativo, la proyección gráfica sobre la cual se puede situar las acciones médicas (toma de remedio, visita al ambulatorio, etc.) parece más fácilmente inteligible por la mayoría de las comunidades cuando se representan los principales momentos de las intervenciones médicas en relación con los símbolos del sol o de la luna (sol saliendo, a la vertical, poniéndose; o bien luna creciente, llena, nueva, decreciente, nueva, etc.). Sin embargo, estos símbolos tienen que ser proyectados sobre curvas, lo que evoca la bóveda celeste. Es, por lo menos, lo que resultó claramente de las consultas y de los esbozos realizados durante los talleres de dibujo organizados para ilustrar los textos y las partes más complejas del mensaje. Por ejemplo, en el caso del complejo y largo tratamiento de la tubercu- 
losis, es importante que el personal médico pueda mostrar al enfermo o a sus padres una representación dibujada de la duración del tratamiento (ver ejemplos de dibujo en J. Chiappino 1997: 274-286).

La comprensión del seguimiento del tratamiento a largo plazo así como el cambio de remedio constituye una dificultad particular. La necesidad de seguir la toma de remedios a pesar de que los signos clínicos desaparecieron a consecuencia del primer efecto del tratamiento, es un obstáculo muy grande en el tratamiento de las endemias más graves como la tuberculosis. Igualmente, es problemático el cambio de remedios en caso de que los primeros no resulten eficaces.

La comparación con la secuencia, tal vez muy larga, de las múltiples intervenciones, además realizadas por varios chamanes, en contra de los agentes patógenos más difíciles de desalojar-chamánicamente- del cuerpo del enfermo, logra ayudar en la comprensión de la duración del tratamiento y del cambio de terapia. En estos casos, la asociación con la actividad chamánica es muy favorable pues ambas estrategias son comparables y se complementan. Es decir, el beneficio que se puede esperar de no desacreditar la actividad chamánica y de poder asociar ambas prácticas para facilitar el entendimiento de las estrategias biomédicas.

\section{Adaptación específica de la presentación de los folletos}

Las ilustraciones son capitales en la producción de los folletos como soporte de formación sanitaria de las comunidades. En nuestro caso multiplicamos los dibujos que fueron realizados por representantes de las comunidades en el transcurso de talleres específicos (ver C. Alès 1997).

Si la ilustración parece indispensable para dar un aspecto más atractivo a los folletos y aclarar unas técnicas bastante complejas, la realización de los dibujos por personas de la cultura presenta un interés de una importancia muy particular. Permite no sólo descubrir malentendidos del mensaje, no anotados a través de la única comunicación oral, sino también evitar, en la esquematización ilustrativa, la proyección occidental de nuestro modo de representar el espacio y el tiempo. 
La comprensión de diversas escenas para ilustrar el proceso propio de una terapéutica compleja o de un proceso biológico, no se encuentra necesariamente facilitada por la disposición, como ya se mencionó, que consiste en presentar los esbozos en líneas que van de la izquierda a la derecha y de arriba para abajo. Mejor será seguir, en muchos casos, los modelos que los dibujantes utilizan espontáneamente con su "perspectiva en escala» ( C. Alès ibid.) o disponiendo las figuras sucesivas sobre una línea que va, sin interrupción, de la derecha a la izquierda y de la derecha a la izquierda, o de arriba para abajo y al revés (lo que recuerda la manera de escribir de la Grecia antigua o del Etrusco, llamada boustrophedon por imitar el surco dejado por el buey que labra la tierra).

Además, aparece que los indígenas no tienen ninguna dificultad para representar en sus dibujos espontáneos la simultaneidad de ambos tratamientos médico y chamánico. Eso no sólo confirma que no consideran cada medicina como exclusiva de la otra, sino también que esta complementariedad puede ser explotada, lo vimos para el ejemplo de la tuberculosis, para hacer entender la duración del tratamiento biológico y/ o el cambio de remedio.

En nuestra experiencia se observa que la intervención de los propios actores indígenas mediante el trabajo de los dibujantes constituye un paso importante en la estimulación de la participación, ya emprendida mediante la colaboración de los traductores en la adaptación de los textos. Los rasgos culturales introducidos por los ilustradores y encontrados en los vestidos de los personajes y el ambiente socioeconómico, refuerzan el deseo de las comunidades de aceptar el mensaje transmitido por los documentos. Gracias a la conveniencia de las imágenes y la adaptación en idioma indígena de lo ilustrado, el documento resulta muy accesible para cada comunidad y, a dos niveles, pues «habla» tanto a los lectores como a los analfabetos.

Sobre este punto hay que destacar que los mayores, aunque muchas veces no leen, miran los folletos con mucha atención e interés. Eso demuestra que los documentos pueden incluso servir para enseñar e incitar a la lectura. La idea según la cual la manera escrita de presentar el mensaje médico podía provocar un rechazo de parte de la población de las 
comunidades que se encuentran en su mayoría analfabetas, estaba muy equivocada. La valorización de la cultura que revela la presentación bajo la forma de consejos sencillos, reunidos en folletos comparables a los libros de los extranjeros y, además, escritos en lengua autóctona, suscitó de inmediato el interés de los indígenas hacia esta producción. Su contenido representa un mensaje accesible en unos documentos propios en los cuales pueden, según dijeron, aprender mucho sobre las enfermedades y sus tratamientos y, finalmente, sobre una cuestión tan preocupante como lo es la cuestión sanitaria.

La diversidad del modo de transcribir los idiomas, conforme a la multitud de los alfabetos introducidos por la variedad del personal educativo, constituye, con las variaciones dialectales existentes entre las regiones, una dificultad que obstaculizó la reivindicación en producir documentos realmente específicos a cada caso. Por límites del tiempo reservado al programa se decidió, para facilitar una más rápida difusión del conocimiento sanitario, publicar los documentos, siguiendo el modo de transcribir mayoritariamente conocido en cada etnia. Esta dificultad debería ser muy fácilmente traspasada según la idea de que lo más importante no es tanto la producción en sí de documentos escritos, sino la transmisión oral de su contenido. Con este propósito, en ciertos casos introdujimos entre comillas unos equivalentes dialectales. Planificamos acompañar la producción de folletos con la grabación de cintas, cuyo modo oral de transmisión, muy factible en las comunidades cercanas de las misiones, podía compensar la dificultad de lectura cualquiera sea su origen ${ }^{10}$.

Sería importante formalizar la integración de folletos bilingües en un plan de formación de promotores o agentes sanitarios indígenas, así como proseguir organizando talleres del tipo de los que permitieron su elaboración pues constituyen elementos importantes en la educación del personal sanitario indígena y la promoción de la higiene entre las poblaciones. Se espera también que tales documentos sean incorporados al currículum escolar a través del Ministerio de Educación en el marco del Régimen de Educación Intercultural Bilingüe ${ }^{11}$. En el futuro se puede proponer no sólo adaptar las escrituras que no convienen para unas comunidades sino también mejorar el estilo mismo. Los folletos produci- 
dos no deben considerarse como hechos de una vez por todas. Se necesita como mínimo pensar en mejorarlos y aportar en los tratamientos que siempre evolucionan las adaptaciones idóneas.

Para concluir insistiremos sobre la importancia del papel educativo para la obtención de la participación de las comunidades en el control de la salud. En este sentido, la producción de material didáctico inteligible es capital. Esto implica como condiciones indispensables que la expresión del mensaje médico y su presentación, bajo la forma de folletos de referencia, sean también redactados en lengua vernácula y sigan conformes a la lógica tradicional. La valorización de la lengua materna y el respeto de las costumbres autóctonas constituyen, con la integración de un personal auxiliar indígena, unos factores inconmensurables en la aceptación de las recomendaciones curativas y profilácticas por las comunidades y la eficacia de todo tipo de servicio médico-asistencial que promover en las situaciones de aislamiento y distancia socioeconómica comparables.

\section{NotAS:}

${ }^{1}$ Diferentes trabajos y programas analizaron los elementos para promover un mejoramiento de la salud en el Amazonas (ver Seijas 1998, Diaz \& Fischer 1993).

${ }^{2}$ Coordinado por el autor, este programa se desarrolló de 1994 a 1997 y fue financiado principalmente por la Comisión de la Comunidad Europea. Se benefició de la participación del Instituto Francés de Investigación Científica para el Desarrollo en Cooperación (ORSTOM-Venezuela), de la Escuela de Antropología de la Facultad de Ciencias Económicas y Sociales (FACES) y del Instituto de Medicina Tropical de la Universidad Central de Venezuela (UCV, Caracas), de las Direcciones Regionales de Salud y de Saneamiento del Ambiente (Puerto Ayacucho), del Servicio Autónomo para el Desarrollo Controlado del Estado Amazonas (SADAAmazonas) del Ministerio del Ambiente y de los Recursos Naturales Renovables (MARNR, Puerto Ayacucho), de la Organización Regional de los Pueblos Indígenas del Amazonas (ORPIA, Puerto Ayacucho),y de la Deutsche Gesellschaft für Technische Zusammenarbeit (G.T.Z., Caracas). Para los resultados del programa, ver J. Chiappino \& C. Alès eds. 1997.

${ }^{3}$ Los proyectos educativos ya propuestos en Venezuela y otros países son generalmente demasiado influenciados por la lógica de la medicina occidental y el modelo 
hospitalo-curativo que ella sigue para ser eficaces en las comunidades aisladas. Ver, por ejemplo, los ensayos de la Fundación Zumaque (1994a, 1994b, 1995), del Ministerio de Salud Pública de la República de Ecuador (1991), de Nicaragua (Vásquez \& Mosquera 1994), de Brasil (Figueroa \& Mainburg 1993) y la crítica del modelo hospitalo-curativo de la medicina asistencial en el Amazonas Venezolano (Armada 1997: 301).

4 "Cómo mantener la salud de su cuerpo», «El agua de beber», «Las diarreas infantiles», "La malaria», «La tuberculosis», «Las infecciones respiratorias agudas infantiles», «Las enfermedades sexualmente transmisibles y el Sida» (Chiappino J. \& C. Alès eds. 1996).

${ }^{5}$ Se organizaron talleres pluriétnicos a Puerto Ayacucho, para las poblaciones indígenas del Norte del estado Amazonas, y a La Esmeralda, para las del Sur.

${ }^{6}$ Pensamos en la creación de palabras que se necesitan introducir para traducir en lenguas amerindias conceptos occidentales como, por ejemplo, los términos inherentes a una Constitución, ver J. Landaburu (en este volumen) para el caso de la traducción en lenguas indígenas de la nueva Constitución de Colombia de 1991.

${ }^{7}$ Sobre la concepción amerindia del equilibrio vital en relación con un balance de caliente/ frío, seco/húmedo, dulce/salado, ver A. Butt Colson \& C. Armellada de 1977, 1985; J. Clarac de Briceño 1981.

${ }^{8}$ Ver UNICEF 1994.

${ }^{9}$ En Venezuela la palabra «té» sirve para definir todo tipo de infusiones preparadas a base de hierbas.

${ }^{10}$ Se grabó una versión sobre casete de los 7 folletos en Yanomami (Chiappino 1998). Fue distribuida a las comunidades satélites de las misiones presentes en el territorio yanomami.

${ }^{11}$ Sobre el Régimen de Educación Intercultural Bilingüe (REIB) en Venezuela, ver M. E. Villalón 1994.

\section{REFERENCIAS:}

Alès, C. (1997) «La vida, la enfermedad y la muerte. Una iconografía de los pueblos de Amazonas», in J. Chiappino \& C. Alès, eds., Del microscopio a la maraca. Caracas: Editorial Ex Libris: 45-224.

Alès, C. \& J. Chiappino (1985a) «Medical Aid, Shamanism and Acculturation among the Yanomami of Venezuela», ARC/IWGIA/SI 53: 73-90.

(1985b) «Chamanisme et médecine: rationalité divergente ou complémentaire? Le problème de l'intégration de l'assistance médicale chez les Yanomami», en Bulletin d'Ethnomédecine, 34: 23-48. 


\section{Jean Chiappino}

Armada, F. (1997) «Aproximación a la situación sanitaria del estado Amazonas», in J Chiappino \& C. Alès, eds., Del microscopio a la maraca. Caracas: Editorial Ex Libris: 293-316

Butt Colson, A. \& C. de Armellada (1977) «Oposiciones binarias y tratamiento de las enfermedades entre los Akawaio», Revista Montalbán, UCAB (6): 183-219.

(1985) «El origen de la etiología de enfermedades y su tratamiento en la América latina» Revista Montalbán, UCAB (16): 5-45.

Chiappino, J. (1992) «Participation caritative ou concertation: alternatives de l'assistance sanitaire prodiguée aux Yanomami du Venezuela», Cahiers de l'ORSTOM, série Sciences Humaines, «Anthropologie et santé publique», vol. 28, no 1: 99-121.

(1995a) "Corps matériel, chamanisme et modernité chez les Yanomami», Techniques et Culture 25-26: 159-192.

(1995b) «El Coloso yanomami frente al nuevo El dorado. Representaciones del ser humano y del medio ambiente: un envite de la participación comunitaria al desarrollo regional», en Amazonas, Modernidad en Tradición, GTZ / SADA-AMAZONAS-CAIAH. Caracas: 175-204.

(1997) «Las Piedras celestes. Para una nueva forma de intercambio en el ámbito de la salud", en J. Chiappino \& C. Alès, eds., Del microscopio a la maraca. Caracas: Editorial Ex Libris: 253-292.

(1998) Palabras para promover la salud entre los pueblos amerindios de Venezuela. Documentos de Educación Sanitaria en Yanomamï. Puerto Ayacucho: Unión Europea / MARNR, Cintas n ${ }^{\circ} 1-3$.

Chiappino, J. \& C. Alès, eds. (1996) Palabras para promover la salud entre los pueblos amerindios de Venezuela. Documentos $n^{\circ} 1$ a 7, 6 volúmenes, versión Chase-, Kurripako-, Wothïha-, Ye'kwana-, Hiwi-, Yanomami-Castellano. Caracas: CCE, PROCOMSIVA.

(1997) Del microscopio a la maraca. Caracas: Editorial Ex Libris, 400 pp.

Clarac de Briceño, J. (1981) Dioses en Exilio. Representaciones y prácticas simbólicas en la Cordillera de Mérida. Caracas: Colección Rescate 2, Fundarte, 259 pp.

Díaz, M. \& J. M. Ficher (1993) «Elementos de análisis para el mejoramiento de la prestación de salud en el Amazona», en E. Estrella \& A. Crespo, eds., Memorias del $1{ }^{\circ}$ Simposio Salud y Población Indígena de la Amazonia, Vol I. Quito: IMPRETEC: 291-295.

Figueroa, A. L. \& E. M. T. Mainburg (1993) «Formação de Indígenas como Agentes Comunitarios no Alto Río Negro, in Salud y Población Indígena de la Amazonia», en ibidem: 243-259.

Fundación Zumaque (1994a) Salud integral. La tuberculosis. Caracas: CIBEARTE C.A. (1994b) Lactancia natural. Alimentos sin igual. Guía para la promoción de la lactancia natural. Caracas: Instituto Nacional de Nutrición, 105 pp.

(1995) Salud integral. Parasitosis intestina. Caracas: CIBERARTE C.A. INA.

OCEI (1993) Censo Indígena de 1992. Vol. I. Caracas: OCEI, Presidencia de la República. República de Ecuador, Ministerio de Salud Pública (1991) Manual de normas para el 
control de enfermedades diarreicas y manejo clínico del paciente con cólera. Quito: Ministerio de Salud Pública, Dirección Nacional de Fomento y Protección, División Nacional de Salud Materno Infantil, Programa Nacional de Control de Enfermedades Diarreicas, 106 pp.

República de Venezuela, Ministerio de Sanidad y Asistencia Social (1991) Manual Normativo para Auxiliares de Medicina Simplificada y otro Personal Voluntario. Caracas: MAS, 319.

Seijas, H. (1988) Informe del Seminario Taller «Aspectos antropológicos y sociológicos de la Atención Primaria en Salud en el Territorio Federal Amazonas", mecanografiado, CAICET, Puerto Ayacucho.

UNICEF (1994) Diagnóstico del Niño Amazónico. Caracas: UNICEF, Corpográfica SRL, $243 \mathrm{pp}$.

Vásquez, M. L. \& M. Mosquera, eds. (1994) Diarrea y Enfermedades Respiratorias Agudas en Nicaragua. Un estudio de Intervención Educativa 1982-1992. Managua: Universidad Centro Americana de Managua, 235 pp.

Villalón, M. E. (1994) Educación para Indígenas en Venezuela: una crítica razonada. Caracas: CEVIAP, Documento de Trabajo n ${ }^{\circ}$ 9, 48 pp. 